\title{
ON FIBRATIONS WHOSE GEOMETRIC FIBERS ARE NONREDUCED
}

\author{
STEFAN SCHRÖER
}

\begin{abstract}
We give a bound on embedding dimensions of geometric generic fibers in terms of the dimension of the base, for fibrations in positive characteristic. This generalizes the well-known fact that for fibrations over curves, the geometric generic fiber is reduced. We illustrate our results with Fermat hypersurfaces and genus 1 curves.
\end{abstract}

\section{$\S 0$. Introduction}

The goal of this article is to understand geometric nonreducedness for fibrations in characteristic $p>0$. Roughly speaking, we deal with nonreducedness that becomes visible only after purely inseparable base change.

My starting point is the following well-known fact: let $k$ be an algebraically closed ground field, let $S$ be a normal algebraic scheme, and let $f: S \rightarrow C$ be a fibration over a curve; then the geometric generic fiber $S_{\bar{\eta}}$ is reduced. This fact goes back to MacLane [18, Theorem 2]; a proof can also be found in Bădescu's monograph [2, Lemma 7.2]. It follows, for example, that geometric nonreducedness plays no role in the Enriques classification of surfaces. A natural question comes to mind: are there generalizations to higher dimensions? The main result of this article is indeed such a generalization.

Theorem. Let $f: X \rightarrow B$ be a proper morphism with $\mathcal{O}_{B}=f_{*}\left(\mathcal{O}_{X}\right)$ between normal $k$-schemes of finite type. Then the generic embedding dimension of $X_{\bar{\eta}}$ is smaller than $\operatorname{dim}(B)$.

Here the generic embedding dimension is the embedding dimension of the local ring at the generic point of $X_{\bar{\eta}}$.

I expect that the phenomenon of geometric nonreducedness in fibrations will play a role in the characteristic $p$-theory of genus 1 fibrations, Albanese

Received May 31, 2009. Accepted February 14, 2010.

2000 Mathematics Subject Classification. Primary 14A15, 14D06, 14J70.

(C) 2011 by The Editorial Board of the Nagoya Mathematical Journal 
maps, and the minimal model program. For example, Kollár [16, Example 4.12] mentions the threefold $X \subset \mathbb{P}^{2} \times \mathbb{P}^{2}$ in characteristic 2 defined by the bihomogeneous equation $x u^{2}+y v^{2}+z w^{2}=0$, whose projection onto the first factor defines a Mori fiber space that has the structure of a geometrically nonreduced conic bundle. The whole theory, however, is largely unexplored to date.

It is actually better to formulate our result in a more general framework, in which an arbitrary field $K$ takes over the role of the function field of the scheme $B$. In this setting, the dimension of $B$, which is also the transcendence degree of the function field $\kappa(B)$, has to be replaced by the degree of imperfection of $K$, which is the length of any $p$-basis for the extension $K^{p} \subset K$.

THEOREM. Let $X$ be a proper normal $K$-scheme with $K=H^{0}\left(X, \mathcal{O}_{X}\right)$. Suppose that $X$ is geometrically nonreduced. Then the geometric generic embedding dimension of $X$ is smaller than the degree of imperfection of $K$.

The key observation of this article is that base change with field extensions $K \subset L$ of degree $p$ cannot produce nilpotent functions on $X \otimes_{K} L$. However, the global functions on the normalization of $X \otimes_{K} L$ may form a field that is larger than $L$. Our arguments also hinge on Kraft's [17] beautiful description of finitely generated field extensions in positive characteristics.

To illustrate our result, we study $p$-Fermat hypersurfaces $X \subset \mathbb{P}_{K}^{n}$, which are defined by a homogeneous equation of the form

$$
\lambda_{0} U_{0}^{p}+\lambda_{1} U_{1}^{p}+\cdots+\lambda_{n} U_{n}^{p}=0
$$

These are twisted forms of an infinitesimal neighborhood of a hyperplane. Mori and Saito [21] studied them in the context of fibrations, using the name wild hypersurface bundle, in connection with the minimal model program. Hoffmann [13] studied such forms from an algebraic perspective, viewing them as generalizations of quadratic forms in characteristic 2; the latter were studied, for example, in [14]. It might be fruitful to combine algebraic and geometric approaches.

We attach to such $p$-Fermat hypersurfaces a numerical invariant $0 \leq d \leq n$ defined as the $p$-degree of certain field extensions depending on the coefficients $\lambda_{i}$ (for details, see Section 3 ), and we show that this number has a geometric significance. 
THEOREM. The p-Fermat hypersurface $X \subset \mathbb{P}^{n}$ is regular if and only if $d=n$. If $X$ is not regular, then the singular set $\operatorname{Sing}(X) \subset X$ has codimension $0 \leq d \leq n-1$.

This generalizes results of Buchweitz, Eisenbud, and Herzog [7, Theorem 1.1], Ahmad [1], and Totaro [23, Lemma 6.6], who studied quadrics in characteristic 2. Our proof uses different methods and relies on Grothendieck's theory of the generic hyperplane section. The result nicely shows that field extensions of degree $p$ may increase the dimension of the singular set only stepwise.

Then we take a closer look at the case of $p$-Fermat plane curves $X \subset \mathbb{P}^{2}$ that have an isolated singularity. It turns out that the normalization is a projective line over a purely inseparable field extension $K \subset L$ of degree $p$. Roughly speaking, the curve $X$ arises from this projective line by thinning out an infinitesimal neighborhood of an $L$-rational point. Here some amazing phenomena related to nonuniqueness of coefficient fields in local rings come to light.

We then study the question of whether curves $C$ arising as such a denormalization are necessarily $p$-Fermat plane curves, which remains unclear. However, we see that it is true under the assumption that $p \neq 3$ and that $C$ globally embeds into a smooth surface. In this context, we touch upon the theory of abstract multiple curves, which were studied, for example, by Bănică and Forster [3], Manolache [19], and Drézet [8]. We also make use of Grothendieck's theory of Brauer-Severi schemes.

Finally, we study genus 1 curves, that is, proper curves $X$ with cohomological invariants $h^{0}\left(\mathcal{O}_{X}\right)=h^{1}\left(\mathcal{O}_{X}\right)=1$. They should play an important role in the characteristic $p$-theory of genus 1 fibrations. Here we have a result on the structure of the Picard scheme: if $X$ is a genus 1 curve that is regular but geometrically nonreduced, then $\operatorname{Pic}_{X}^{0}$ is unipotent, that is, a twisted form of the additive group scheme $\mathbb{G}_{a}$; as a consequence, the reduction of $X \otimes_{K} \bar{K}$ cannot be an elliptic curve.

\section{$\S 1$. Geometric nonreducedness}

Throughout this article, we fix a prime number $p>0$. Let $K$ be a field of characteristic $p$, and let $X$ be a proper $K$-scheme so that the canonical inclusion $K \subset H^{0}\left(X, \mathcal{O}_{X}\right)$ is bijective. The latter can always be achieved, at least if $X$ is connected and reduced, by replacing the ground field by the finite extension field $H^{0}\left(X, \mathcal{O}_{X}\right)$. If $K \subset K^{\prime}$ is a field extension, the 
induced proper $K^{\prime}$-scheme $X^{\prime}=X \otimes_{K} K^{\prime}$ may be less regular than $X$, and in fact nilpotent elements may appear. Such phenomena are the topic of this article. We start with obvious facts.

Proposition 1.1. If $X$ contains no embedded components, then $X^{\prime}=$ $X \otimes_{K} K^{\prime}$ contains no embedded components. In particular, if $X$ is reduced, then $X^{\prime}$ is reduced if and only if $X^{\prime}$ is generically reduced.

Proof. The first statement is contained in [9, proposition 4.2.7], and the second is an immediate consequence.

Recall that $X$ is called geometrically reduced if $X^{\prime}=X \otimes_{K} K^{\prime}$ is reduced for all field extensions $K \subset K^{\prime}$. Actually, it suffices to check reducedness for a single field extension.

Proposition 1.2. The scheme $X$ is geometrically reduced if and only if $X \otimes_{K} K^{1 / p}$ is reduced.

Proof. Necessity is trivial. Now suppose that $X \otimes_{K} K^{1 / p}$ is reduced. Let $\eta \in X$ be a generic point, and let $F=\mathcal{O}_{X, \eta}$ be the corresponding field of functions. Then $F \otimes_{K} K^{1 / p}$ is reduced. By MacLane's criterion (see, e.g., [6, Chapter V, Section 15, No. 4, Theorem 2]), the field extension $K \subset F$ is separable. In light of Proposition 1.1, $X$ must be geometrically reduced.

Purely inseparable field extensions that are smaller than $K^{1 / p}$ do not necessarily uncover geometric nonreducedness. In fact, degree $p$ extensions are incapable of doing so.

Lemma 1.3. Suppose that $K \subset K^{\prime}$ is purely inseparable of degree $p$. If the scheme $X$ is normal, then the induced scheme $X^{\prime}=X \otimes_{K} K^{\prime}$ is at least integral.

Proof. Let $F=\mathcal{O}_{X, \eta}$ be the function field of $X$. In light of Proposition 1.1, it suffices to check that the local Artin ring $F^{\prime}=F \otimes_{K} K^{\prime}$ is a field. Choose an element $a \in K^{\prime}$ not contained in $K$. Then $b=a^{p}$ lies in the subfield $K \subset K^{\prime}$, and $T^{p}-b \in K[T]$ is the minimal polynomial of $a \in K^{\prime}$. It follows that $K^{\prime}=K[T] /\left(T^{p}-b\right)$ and $F^{\prime}=F[T] /\left(T^{p}-b\right)$. Thus, our task is to show that $b \in F$ is not a $p$ th power. Seeking a contradiction, we assume that $b=c^{p}$ for some $c \in F$. Then, for each affine open subset $U=\operatorname{Spec}(A)$ in $X$, the element $c \in F$ lies in the integral closure of $A \subset F$. Since $X$ is normal, $c \in A$, whence $c \in H^{0}\left(X, \mathcal{O}_{X}\right)$ according to the sheaf axioms. By our overall assumption, $K=H^{0}\left(X, \mathcal{O}_{X}\right)$, and we conclude that $b$ is a $p$ th power in $K$, a contradiction. 
Suppose that our field extension $K \subset K^{\prime}$ is such that the induced scheme $X^{\prime}=X \otimes_{K} K^{\prime}$ remains integral; then we consider its normalization $Z \rightarrow X^{\prime}$ and obtain a new field $L=H^{0}\left(Z, \mathcal{O}_{Z}\right)$ such that we have a sequence of field extensions

$$
K \subset K^{\prime} \subset L
$$

Here $K^{\prime} \subset L$ is finite. In some sense and under suitable assumptions, this extension $K \subset L$ is also not too big. Recall that a purely inseparable field extension $K \subset E$ is called of height at most 1 if one has $E^{p} \subset K$.

Proposition 1.4. Suppose that $K \subset K^{\prime}$ is purely inseparable of height at most 1 so that $X^{\prime}=X \otimes_{K} K^{\prime}$ is integral. Then the field extension $K \subset L$ is purely inseparable and has height at most 1 as well.

Proof. Let $f \in H^{0}\left(Z, \mathcal{O}_{Z}\right)$. We have to show that $g=f^{p}$ lies in the image of $H^{0}\left(X, \mathcal{O}_{X}\right)$ with respect to the canonical projection $Z \rightarrow X$. Let $F$ and $F^{\prime}$ be the function fields of $X$ and $X^{\prime}$, respectively. Then $F^{\prime}=F \otimes_{K} K^{\prime}$, and this is also the function field of $Z$. The description of $F^{\prime}$ as tensor product gives $g \in F$. To finish the proof, let $x \in X$ be a point of codimension 1 . Using the fact that $X$ is normal, it suffices to show that $g \in F$ is contained in the valuation ring $\mathcal{O}_{X, x} \subset F$. Suppose that this is not the case. Then $1 / g \in \mathfrak{m}_{x}$. Consider the point $z \in Z$ corresponding to $x \in X$. Then $1 / g \in \mathfrak{m}_{z}$ since $\mathfrak{m}_{z} \cap \mathcal{O}_{X, x}=\mathfrak{m}_{x}$. On the other hand, we have $g \in \mathcal{O}_{Z, z}$, whence $1 \in \mathfrak{m}_{z}$, a contradiction.

Recall that the $p$-degree of an extension $K \subset L$ of height at most 1 is the cardinality of any $p$-basis (see [6, Chapter V, Section 13]). The $p$-degree $[L: K]_{p}$ of an arbitrary extension is defined as the $p$-degree of the height 1 extension $K\left(L^{p}\right) \subset L$. The degree of imperfection of a field $K$ is the $p$-degree of $K$ over its prime field. In other words, it is the $p$-degree of $K^{p} \subset K$ or, equivalently, of $K \subset K^{1 / p}$.

Proposition 1.5. There is a purely inseparable extension $K \subset K^{\prime}$ of height at most 1 so that the induced scheme $X^{\prime}=X \otimes_{K} K^{\prime}$ is integral and so that the field of global functions $L=H^{0}\left(Z, \mathcal{O}_{Z}\right)$ on the normalization $Z \rightarrow X^{\prime}$ is isomorphic to $K^{1 / p}$ as an extension of $K$.

Proof. This is an application of Zorn's lemma. Let $K \subset K_{\alpha} \subset K^{1 / p}$ be the collection of all intermediate fields with $X \otimes_{K} K_{\alpha}$ integral. We may view this collection as an ordered set, where the ordering comes from the inclusion relation. This ordered set is inductive by [10, corollaire 8.7.3]. 
By Zorn's lemma, we choose a maximal intermediate field $K^{\prime}=K_{\alpha}$, and we consider $L=H^{0}\left(Z, \mathcal{O}_{Z}\right)$. Then $L \subset K^{1 / p}$ by Proposition 1.4. Seeking a contradiction, we assume that the latter inclusion is not an equality. Then there is a purely inseparable extension $K \subset E$ of degree $p$ that is linearly disjoint from $K \subset L$. Consider the composite field $K^{\prime \prime}=K^{\prime} \otimes_{K} E$. Then $K \subset K^{\prime \prime}$ is purely inseparable of height at most 1 and strictly larger than $K^{\prime}$, so $X^{\prime \prime}=X \otimes_{K} K^{\prime \prime}$ is generically nonreduced. On the other hand, $X^{\prime \prime}$ is birational to

$$
Z \otimes_{K^{\prime}} K^{\prime \prime}=Z \otimes_{L}\left(L \otimes_{K^{\prime}} K^{\prime \prime}\right)=Z \otimes_{L}\left(L \otimes_{K} E\right),
$$

which is integral by Lemma 1.3, a contradiction.

\section{§2. Generic embedding dimension}

Let $K$ be a field of characteristic $p>0$, and let $K \subset F$ be a finitely generated extension field. If $K \subset K^{\prime}$ is purely inseparable, the tensor product $R=F \otimes_{K} K^{\prime}$ is a local Artin ring. We now investigate its embedding dimension $\operatorname{edim}(R)$, that is, the smallest number of generators for the maximal ideal $\mathfrak{m}_{R}$ or, equivalently, the vector space dimension of the cotangent space $\mathfrak{m}_{R} / \mathfrak{m}_{R}^{2}$ over the residue field $R / \mathfrak{m}_{R}$.

We first will relate the embedding dimension edim $\left(F \otimes_{K} K^{\prime}\right)$, which we regard as an invariant from algebraic geometry, with some invariants from field theory. Our analysis hinges on Kraft's beautiful result on the structure of finitely generated field extensions. According to [17], there is a chain of intermediate fields

$$
K \subset F_{0} \subset F_{1} \subset \cdots \subset F_{m}=F
$$

so that $K \subset F_{0}$ is separable, each $F_{i} \subset F_{i+1}$ is purely inseparable, and moreover, $F_{i+1}$ is generated over $F_{i}$ by a single element $a_{i}$ whose minimal polynomial is of the form $T^{p^{r_{i}}}-b_{i}$ with constant term $b_{i} \in K\left(F_{i}^{p^{r_{i}}}\right)$ and $r_{i}>0$. We now exploit the rather special form of the constant terms $b_{i}$.

Proposition 2.1. Suppose that the extension $K \subset K^{\prime}$ contains $K^{1 / p}$. Then the following integers coincide:

(i) the embedding dimension of $F \otimes_{K} K^{\prime}$;

(ii) the number $m$ of purely inseparable field extensions in the chain (1);

(iii) the difference between the p-degree and the transcendence degree of $K \subset F$. 
Proof. Recall that the $p$-degree of an arbitrary field extension $K \subset F$ is defined as the $p$-degree of the height $\leq 1$ extension $K\left(F^{p}\right) \subset F$. Let $t_{1}, \ldots, t_{n} \in F_{0}$ be a separating transcendence basis over $K$, such that $n$ is the transcendence degree for $K \subset F$. Clearly, $t_{1}, \ldots, t_{n}$ together with $a_{1}, \ldots, a_{m}$ compose a $p$-basis for $K\left(F^{p}\right) \subset F$, so the integers in (ii) and (iii) are indeed the same.

We now check that $\operatorname{edim}\left(F \otimes_{K} K^{\prime}\right)=m$ by induction on $m$. If $m=0$, then $K \subset F$ is separable, whence $F \otimes_{K} K^{\prime}$ is a field. Suppose now that $m \geq 1$, and assume inductively that the local Artin ring $F_{m-1} \otimes_{K} K^{\prime}$ has embedding dimension $m-1$. Write $F=F_{m-1}[T] /\left(T^{p^{r}}-b\right)$ for some $b \in$ $K\left(F_{m-1}^{p^{r}}\right)$. Clearly, $b$ is not a $p$ th power, but it becomes a $p$ th power after tensoring with $K^{\prime}$ because $K^{1 / p} \subset K^{\prime}$. Now write

$$
F \otimes_{K} K^{\prime}=\left(F_{m-1} \otimes_{K} K^{\prime}\right)[T] /\left(T^{p^{r}}-b \otimes 1\right) .
$$

Our claim then follows from the following lemma.

Lemma 2.2. Suppose that $R$ is a local Noetherian ring in characteristic $p>0$, and let $A=R[T] /\left(T^{p^{r}}-f^{p}\right)$ for some integer $r \geq 1$ and some element $f \in R$. Then we have $\operatorname{edim}(A)=\operatorname{edim}(R)+1$.

Proof. We may assume that $\mathfrak{m}_{R}^{2}=0$. Let $\bar{f}$ denote the class of $f$ in the residue field $k=R / \mathfrak{m}_{R}$. Write $\bar{f}=\bar{g}^{p^{m-1}}$ with $\bar{g} \in k$ and $0 \leq m-1 \leq r$ as large as possible, and choose a representant $g \in R$ for $\bar{g}$. Then $f^{p}-g^{p^{m}} \in \mathfrak{m}_{R}^{p}$, whence $f^{p}=g^{p^{m}}$. Set $h=T^{p^{r-m}}-g$, such that $A=R[T] /\left(h^{p^{m}}\right)$. Consider the ring $A^{\prime}=R[T] /(h)$. This is a gonflement of $R$ in the sense of $[5$, chapitre IX, appendice], and we have $\operatorname{edim}\left(A^{\prime}\right)=\operatorname{edim}(R)$ by $[5$, chapitre IX, appendice, proposition 2]. The element $h \in A$ is nilpotent, and $A^{\prime}=A / h A$ by definition. It therefore suffices to check that $h \notin \mathfrak{m}_{A}^{2}$. Seeking a contradiction, we assume that $h \in \mathfrak{m}_{A}^{2}$. Applying the functor $\bigcap_{R} k$, we reduce to the case that $R=k$ and $R^{\prime}=k^{\prime}$ are fields such that $\mathfrak{m}_{A}=h A$. It then follows that $\mathfrak{m}_{A}=\bigcap_{i>0} \mathfrak{m}_{A}^{i}=0$ such that the projection $A \rightarrow A^{\prime}$ is bijective. Taking ranks of these free $R$-modules, we obtain $p^{m}=1$ and thus $m=0$, which contradicts $0 \leq m-1$.

In [17], the integer in Proposition 2.1 is called the inseparability of $K \subset F$. We prefer to call it the geometric embedding dimension for $K \subset F$, to avoid confusion with other measures of inseparability and to stress its geometric meaning. Note that this invariant depends neither on the choice of $K^{\prime}$ nor on the choice of the particular chain (1). 
We now go back to the setting of algebraic geometry. Suppose that $X$ is an integral proper $K$-scheme with $K=H^{0}\left(X, \mathcal{O}_{X}\right)$. Let $F=\mathcal{O}_{X, \eta}$ be its function field. We call the embedding dimension of $F \otimes_{K} K^{1 / p}$ the geometric generic embedding dimension of $X$. The main result of the article is the following.

TheOREM 2.3. Let $X$ be a proper normal $K$-scheme with $K=H^{0}(X$, $\left.\mathcal{O}_{X}\right)$. Suppose that $X$ is not geometrically reduced. Then the geometric generic embedding dimension of $X$ is smaller than the degree of imperfection for $K$.

Proof. The statement is trivial if the degree of imperfection for $K$ is infinite. Assume now that this degree of imperfection is finite. According to Proposition 1.5, there is a purely inseparable extension $K \subset K^{\prime}$ of height $\leq 1$ so that the induced scheme $X^{\prime}=X \otimes_{K} K^{\prime}$ is integral and that its normalization $Z$ has the property that $H^{0}\left(Z, \mathcal{O}_{Z}\right)=K^{1 / p}$. Note that $K$ cannot be perfect, since $X$ is not geometrically reduced, and hence $K \neq K^{\prime}$. We now use the transitivity properties of tensor products. The scheme

$$
X \otimes_{K} K^{1 / p}=\left(X \otimes_{K} K^{\prime}\right) \otimes_{K^{\prime}} K^{1 / p}=X^{\prime} \otimes_{K^{\prime}} K^{1 / p}
$$

is birational to

$$
Z \otimes_{K^{\prime}} K^{1 / p}=Z \otimes_{K^{1 / p}}\left(K^{1 / p} \otimes_{K^{\prime}} K^{1 / p}\right) .
$$

The local Artin ring $K^{1 / p} \otimes_{K^{\prime}} K^{1 / p}$ has residue field $K^{1 / p}$, and its embedding dimension coincides with the $p$-degree of $K^{\prime} \subset K^{1 / p}$, by Lemma 2.6 below. This embedding dimension is clearly the geometric generic embedding dimension of $X$. The $p$-degree of $K^{\prime} \subset K^{1 / p}$ is strictly smaller than the $p$-degree of $K \subset K^{1 / p}$, which coincides with the degree of imperfection for $K$.

Corollary 2.4. Let $k$ be a perfect field, and let $f: X \rightarrow B$ be a proper morphism with $\mathcal{O}_{B}=f_{*}\left(\mathcal{O}_{X}\right)$ between integral normal algebraic $k$-schemes. Let $\eta \in B$ be the generic point, and suppose that $\operatorname{dim}(B)>0$. Then the geometric generic embedding dimension of $X_{\eta}$ is smaller than $\operatorname{dim}(B)$.

Proof. The statement is trivial if $X_{\eta}$ is geometrically reduced. If $X_{\eta}$ is not geometrically reduced, then its geometric generic embedding dimension is smaller than the degree of imperfection of the function field $K=\mathcal{O}_{B, \eta}$. The latter coincides with $\operatorname{dim}(B)$ by $[6$, Chapter V, Section 16 , No. 6, Corollary 2 to Theorem 4], because the ground field $k$ is perfect. 
Let us also restate MacLane's result [18, Theorem 2] in geometric form.

Corollary 2.5. With assumptions as in Corollary 2.4, suppose additionally that $B$ is a curve. Then $X_{\eta}$ is geometrically reduced.

Proof. According to Corollary 2.4, the geometric generic embedding dimension of $X_{\eta}$ is zero.

In the proof for Theorem 2.3, we needed the following fact.

LEMMA 2.6. Let $K \subset L$ be a finite purely inseparable extension of height $\leq 1$. Then the local Artin ring $R=L \otimes_{K} L$ has residue field isomorphic to $L$, and its embedding dimension equals the $p$-degree of $K \subset L$.

Proof. Choose a $p$-basis $a_{1}, \ldots, a_{n} \in L$, say, with $a_{i}^{p}=b_{i} \in K$. Then we have $L=K\left[T_{1}, \ldots, T_{n}\right] /\left(T_{1}^{p}-b_{1}, \ldots, T_{n}^{p}-b_{n}\right)$, and consequently

$$
R=L\left[U_{1}, \ldots, U_{n}\right] /\left(U_{1}^{p}-a_{1}^{p}, \ldots, U_{n}^{p}-a_{n}^{p}\right)
$$

Clearly, the $U_{i}-a_{i}$ are nilpotent and generate the $L$-algebra $R$, and their residue classes in $\mathfrak{m}_{R} / \mathfrak{m}_{R}^{2}$ compose a vector space basis. Hence, $\operatorname{edim}(R)$ equals the $p$-degree of $K \subset L$.

\section{$\S 3 . \quad p$-Fermat hypersurfaces}

Let $K$ be a ground field of characteristic $p>0$. In this section, we shall consider Fermat hypersurfaces $X \subset \mathbb{P}^{n}$ defined by a homogeneous equation of the special form

$$
\lambda_{0} U_{0}^{p}+\lambda_{1} U_{1}^{p}+\cdots+\lambda_{n} U_{n}^{p}=0
$$

Here we write $\mathbb{P}^{n}=\operatorname{Proj}\left(K\left[U_{0}, \ldots, U_{n}\right]\right)$, and $\lambda_{0}, \ldots, \lambda_{n} \in K$ are scalars not all zero. Let us call such subschemes $p$-Fermat hypersurfaces. If the scalars are contained in $K^{p} \subset K$, then $X$ is the $(p-1)$ th infinitesimal neighborhood of a hyperplane. In any case, $X$ is a twisted form of the $(p-1)$ th infinitesimal neighborhood of a hyperplane such that $X$ is nowhere smooth. The following is immediate.

Proposition 3.1. We have $X(K)=\emptyset$ if and only if the scalars $\lambda_{0}, \ldots$, $\lambda_{n} \in K$ are linearly independent over $K^{p}$. 
The main goal of this section is to understand the singular locus $\operatorname{Sing}(X) \subset X$, which comprises the points $x \in X$ whose local ring $\mathcal{O}_{X, x}$ is not regular. To this end, let

$$
f=\lambda_{0} U_{0}^{p}+\lambda_{1} U_{1}^{p}+\cdots+\lambda_{n} U_{n}^{p} \in K\left[U_{0}, \ldots, U_{n}\right]
$$

be the homogeneous polynomial defining $X=V_{+}(f)$, and consider the intermediate field

$$
K^{p} \subset E \subset K
$$

generated over $K^{p}$ by all fractions $f\left(\alpha_{0}, \ldots, \alpha_{n}\right) / f\left(\beta_{0}, \ldots, \beta_{n}\right)$ with nonzero denominator and scalars $\alpha_{i}, \beta_{i} \in K$. Clearly, this intermediate field depends only on the closed subscheme $X \subset \mathbb{P}^{n}$ and does not change if one translates $X$ by an automorphism of $\mathbb{P}^{n}$. A more direct but less invariant description of this intermediate field is as follows.

Proposition 3.2. Suppose that $\lambda_{r} \neq 0$. Then the extension $K^{p} \subset E$ is generated by the fractions $\lambda_{i} / \lambda_{r}, 0 \leq i \leq n$.

Proof. Let $K^{p} \subset E^{\prime} \subset K$ be the intermediate field generated by the $\lambda_{i} / \lambda_{r}$. The inclusion $E^{\prime} \subset E$ is trivial. The converse inclusion follows from

$$
f\left(\alpha_{0}, \ldots, \alpha_{n}\right) / f\left(\beta_{0}, \ldots, \beta_{n}\right)=\sum_{i=0}^{n} \alpha_{i}^{p}\left(\sum_{j=0}^{n} \frac{\lambda_{j}}{\lambda_{r}} \frac{\lambda_{r}}{\lambda_{i}} \beta_{j}^{p}\right)^{-1},
$$

where the outer sum is restricted to those indices $0 \leq i \leq n$ with $\lambda_{i} \neq 0$.

We obtain a numerical invariant $d=\left[E: K^{p}\right]_{p}$ for our $p$-Fermat variety $X \subset \mathbb{P}^{n}$, the $p$-degree of the field extension $K^{p} \subset E$. In light of Proposition 3.2 , we have $0 \leq d \leq n$. This numerical invariant has a geometric significance as follows.

THEOREM 3.3. The scheme $X$ is regular if and only if $d=n$. If the singular set $\operatorname{Sing}(X) \subset X$ is nonempty, then its codimension equals the p-degree $d=\left[E: K^{p}\right]_{p}$.

Proof. Let $c \in\{0, \ldots, n-1, \infty\}$ be the codimension of $\operatorname{Sing}(X) \subset X$. In the first part of the proof we show that $c \geq d$. By convention, the case $c=\infty$ means that $\operatorname{Sing}(X)$ is empty. Without loss of generality, we may assume that $\lambda_{0}=1$ and that $\lambda_{1}, \ldots, \lambda_{d} \in K$ are $p$-linearly independent. For $d+1 \leq j \leq n$, we then may write

$$
\lambda_{j}=P_{j}\left(\lambda_{1}, \ldots, \lambda_{d}\right),
$$


where $P_{j}\left(V_{1}, \ldots, V_{d}\right)$ is a polynomial with coefficients in $K^{p}$ and of degree $\leq p-1$ in each of the variables. Since the scalars $\lambda_{1}, \ldots, \lambda_{d} \in K$ are $p$-linearly independent, there are derivations $D_{i}: K \rightarrow K$ with $D_{i}\left(\lambda_{j}\right)=\delta_{i j}$, the Kronecker delta. We may extend them to derivations of degree zero $D_{i}: K\left[X_{0}, \ldots, X_{n}\right] \rightarrow K\left[X_{0}, \ldots, X_{n}\right]$, sending the variables to zero. Then the singular locus of $X=V_{+}(f)$ is contained in the closed subscheme $S \subset \mathbb{P}^{n}$ defined by the vanishing of the homogeneous polynomials

$$
\begin{gathered}
f=U_{0}^{p}+\lambda_{1} U_{1}^{p}+\cdots+\lambda_{n} U_{n}^{p} \\
D_{i}(f)=U_{i}^{p}+\sum_{j=d+1}^{n} \frac{\partial P_{j}}{\partial V_{i}}\left(\lambda_{1}, \ldots, \lambda_{d}\right) U_{j}^{p}, \quad 1 \leq i \leq d .
\end{gathered}
$$

Substituting the relations $D_{i}(f)$ into the former relation $f=0$, we infer that $S \subset \mathbb{P}^{n}$ is defined by the vanishing of homogeneous polynomials of the form

$$
U_{i}^{p}-Q_{i}\left(U_{d+1}, \ldots, U_{n}\right), \quad 0 \leq i \leq d
$$

It suffices to check that $\operatorname{dim}(S) \leq n-1-d$. Suppose that this is not the case. By Krull's principal ideal theorem, the dimensions of $S^{\prime}=S \cap V_{+}\left(U_{d+1}, \ldots\right.$, $\left.U_{n}\right)$ are $\geq 0$. On the other hand, we have $S_{\text {red }}^{\prime}=V_{+}\left(U_{0}, \ldots, U_{n}\right)=\emptyset$, a contradiction. This proves that $c \geq d$. In particular, the scheme $X$ is regular provided that $d=n$.

To finish the proof, we claim that $c=d$ if $d<n$. We proof the claim by induction on $n$. The case $n=1$ is trivial. Now suppose that $n \geq 2$ and that the claim is true for $n-1$. Suppose that $d<n$; otherwise, there is nothing to prove. Without loss of generality, we may assume that $\lambda_{n} \in K$ is nonzero and $p$-linearly dependent on $\lambda_{0}, \ldots, \lambda_{n-1}$.

To proceed we employ Grothendieck's theory of the generic hyperplane section as exposed in the unpublished manuscript [12], confer also Jouanolou's monograph [15]. Let $\check{\mathbb{P}}^{n}$ be the scheme of hyperplanes in $\mathbb{P}^{n}$, let $H \subset \mathbb{P}^{n} \times \check{\mathbb{P}}^{n}$ be the universal hyperplane, and let $\eta \in \check{\mathbb{P}}^{n}$ be the generic point. We then denote by $Y=\left(X \times \check{\mathbb{P}}^{n}\right) \cap H$ the universal hyperplane section of $X$, and by $Y_{\eta}=Y \times_{\check{\mathbb{P}}_{n}}$ Spec $\kappa(\eta)$ the generic hyperplane section of $X$. Note that $Y_{\eta}$ is actually a hyperplane section in $X \otimes_{K} \kappa(\eta)$ but that the projection $Y_{\eta} \rightarrow X$ has highly unusual geometric properties.

We have a closed embedding $Y_{\eta} \subset H_{\eta}$, and $H_{\eta}$ is isomorphic to the projective space of dimension $n-1$ over $K^{\prime}=\kappa(\eta)$. To describe it explicitly, let $U_{i}^{*}$ be the homogeneous coordinates for $\check{\mathbb{P}}^{n}$ dual to the $U_{i}$. Then the universal 
hyperplane $H \subset \mathbb{P}^{n} \times \check{\mathbb{P}}^{n}$ is defined by the bihomogeneous equation $\sum U_{i} \otimes$ $U_{i}^{*}=0$, and the function field of $\check{\mathbb{P}}^{n}$ is the subfield $K^{\prime} \subset K\left(U_{0}^{*}, \ldots, U_{n}^{*}\right)$ generated by the fractions $U_{i}^{*} / U_{n}^{*}, 0 \leq i \leq n$. In turn, the generic hyperplane $H_{\eta} \subset \mathbb{P}_{\eta}^{n}$ is defined by the homogeneous equation $\sum_{i=0}^{n} U_{i} \otimes U_{i}^{*} / U_{n}^{*}$ such that we have the additional relation $U_{n} \otimes 1=-\sum_{i=0}^{n-1} U_{i} \otimes U_{i}^{*} / U_{n}^{*}$. Hence, we may write the generic hyperplane $H_{\eta}=\operatorname{Proj} K^{\prime}\left[U_{0}^{\prime}, \ldots, U_{n-1}^{\prime}\right]$ with $U_{i}^{\prime}=U_{i} \otimes 1$. In these homogeneous coordinates, the generic hyperplane section is the $p$-Fermat variety given by $Y_{\eta}=V_{+}\left(f^{\prime}\right)$ with

$$
f^{\prime}=\sum_{i=0}^{n-1}\left(\lambda_{i} / \lambda_{n}-\left(U_{i}^{*} / U_{n}^{*}\right)^{p}\right)\left(U_{i}^{\prime}\right)^{p} .
$$

Now let $c^{\prime} \in\{0, \ldots, n-2, \infty\}$ be the codimension of the singular set $\operatorname{Sing}\left(Y_{\eta}\right) \subset Y_{\eta}$, and let $d^{\prime}=\left[E^{\prime}: K^{\prime p}\right]_{p}$ be the numerical invariant of $p$-Fermat variety $Y_{\eta} \subset H_{\eta}$. Using that $\lambda_{n}$ is $p$-linearly dependent on $\lambda_{1}, \ldots, \lambda_{n-1}$, we easily see that $d=d^{\prime}$. We now use that the projection $Y_{\eta} \rightarrow X$ is flat with geometrically regular fibers and that its set-theoretical image is the set of nonclosed points $x \in X$. This implies that

$$
c^{\prime}= \begin{cases}\infty & \text { if } c=n-1 \\ c & \text { if } c \leq n-2 .\end{cases}
$$

We now distinguish two cases. Suppose first that $d=n-1$, such that $d^{\prime}=$ $n-1$. We already saw that this implies that $Y_{\eta}$ is regular, whence $c^{\prime}=\infty$, and therefore $c=n-1$. Now consider the case that $d \leq n-2$. Then $d^{\prime} \leq$ $(n-1)-1$, and the induction hypothesis implies that $c^{\prime}=d^{\prime}$, and finally $d=d^{\prime}=c^{\prime}=c$. Summing up, we have $c=d$ in both cases.

\section{$\S 4$. Singularities on $p$-Fermat plane curves}

Keeping the assumptions of the previous section, we now study the singularities lying on $p$-Fermat plane curves $X \subset \mathbb{P}_{K}^{2}$, say, defined by

$$
\lambda_{0} U_{0}^{p}+\lambda_{1} U_{1}^{p}+\lambda_{2} U_{2}^{p}=0
$$

with $\lambda_{0}, \lambda_{1}, \lambda_{2} \in K$. First note that $X$ is a geometrically irreducible projective curve and has $h^{0}\left(\mathcal{O}_{X}\right)=1$ and $h^{1}\left(\mathcal{O}_{X}\right)=(p-1)(p-2) / 2$. Let $0 \leq d \leq 2$ be the numerical invariant of the $p$-Fermat variety $X$, as defined in the previous section. The curve $X$ is regular if $d=2$, is nonreduced if $d=0$, and has isolated singularities if $d=1$. 
With respect to singularities, only the case $d=1$ is of interest. Throughout, we suppose that $d=1$. Without loss of generality, we may then assume that $\lambda_{2}=1$, that $\lambda=\lambda_{0}$ does not lie in $K^{p}$, and that $\lambda_{1}=P(\lambda)$ is a polynomial of degree $<p$ in $\lambda$. So $X \subset \mathbb{P}_{K}^{2}$ is defined by the homogeneous equation

$$
\lambda U_{0}^{p}+P(\lambda) U_{1}^{p}+U_{2}^{p}=0
$$

Consider the field extension $L=K\left(\lambda^{1 / p}\right)$, and let $T_{0}, T_{1}$ be two indeterminates. The map

$$
\begin{gathered}
K\left[U_{0}, U_{1}, U_{2}\right] \longrightarrow L\left[T_{0}, T_{1}\right], \\
U_{0} \longmapsto T_{0}, \quad U_{1} \longmapsto T_{1}, \quad U_{2} \longmapsto-\lambda^{1 / p} T_{0}-P\left(\lambda^{1 / p}\right) T_{1}
\end{gathered}
$$

defines a morphism $\nu: \mathbb{P}_{L}^{1} \rightarrow \mathbb{P}_{K}^{2}$ factoring over $X \subset \mathbb{P}_{K}^{2}$.

Proposition 4.1. The induced morphism $\nu: \mathbb{P}_{L}^{1} \rightarrow X$ is the normalization of $X$.

Proof. It suffices to check that the morphism $\nu: \mathbb{P}_{L}^{1} \rightarrow X$ has degree 1 . The intersection $X \cap V_{+}\left(U_{0}\right)$ is a Cartier divisor of length $p$. Its preimage on $\mathbb{P}_{L}^{1}$ is given by $V_{+}\left(T_{0}\right)$, which also has length $p$ over $K$, whence the degree in question is 1 .

It follows that the field extension $K \subset L$ is nothing but the field of global section on the normalization of $X$. Therefore, it depends only on the curve $X$ and not on the chosen Fermat equation (2).

Proposition 4.2. The singular locus $\operatorname{Sing}(X)$ consists of precisely one point $a_{0} \in X$. The corresponding point $a \in \mathbb{P}_{L}^{1}$ on the normalization has residue field $\kappa(a)=L$.

Proof. Taking the derivative with respect to $\lambda$, we see that the singular locus $\operatorname{Sing}(X)$ is contained in the closed subscheme defined by

$$
\lambda U_{0}^{p}+P(\lambda) U_{1}^{p}+U_{2}^{p}=0 \quad \text { and } \quad U_{0}^{p}+P^{\prime}(\lambda) U_{1}^{p}=0 .
$$

Substituting the latter into the former, we see that there is only one singular point $a_{0} \in X$. The preimage on $\mathbb{P}_{L}^{1}$ is defined by $T_{0}^{p}+P^{\prime}(\lambda) T_{1}^{p}$, which clearly defines an $L$-rational point $a \in \mathbb{P}_{L}^{1}$.

REMARK 4.3. The homogeneous coordinates for the preimage $a \in \mathbb{P}_{L}^{1}$ of the singular point $a_{0} \in X$ are $\left(-P^{\prime}\left(\lambda^{1 / p}\right): 1\right)$. 
Let $\mathfrak{c} \subset \nu_{*}\left(\mathcal{O}_{\mathbb{P}_{L}^{1}}\right)$ be the conductor ideal for the finite birational map $\nu: \mathbb{P}_{L}^{1} \rightarrow X$, which is the largest $\nu_{*}\left(\mathcal{O}_{\mathbb{P}_{L}^{1}}\right)$-ideal contained in the subring $\mathcal{O}_{X} \subset \nu_{*}\left(\mathcal{O}_{\mathbb{P}_{L}^{1}}\right)$. The conductor ideal defines closed subschemes $A \subset \mathbb{P}_{L}^{1}$ and $A_{0} \subset X$, such that we have a commutative diagram

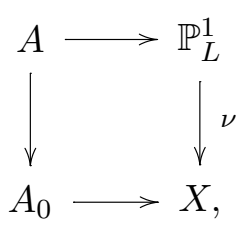

which is Cartesian and co-Cartesian. By abuse of notation, we write $\mathcal{O}_{A_{0}}$ and $\mathcal{O}_{A}$ for the local Artin rings defining the conductor schemes $A_{0}$ and $A$. Then we have $\mathcal{O}_{A}=L[u] /\left(u^{l}\right)$ for some integer $l \geq 1$, where $u \in \mathcal{O}_{\mathbb{P}^{1}, a}$ denotes a uniformizer, and the subring $\mathcal{O}_{A_{0}} \subset \mathcal{O}_{A}$ is a $K$-subalgebra.

Proposition 4.4. We have $\mathcal{O}_{A}=L[u] /\left(u^{p-1}\right)$, and $\mathcal{O}_{A_{0}} \subset \mathcal{O}_{A}$ is a $K$ subalgebra generated by two elements so that $L \not \subset \mathcal{O}_{A_{0}}$ and $\operatorname{dim}_{K}\left(\mathcal{O}_{A_{0}}\right)=$ $p(p-1) / 2$.

Proof. The conductor square (3) yields an exact sequence of coherent sheaves

$$
0 \longrightarrow \mathcal{O}_{X} \longrightarrow \mathcal{O}_{A_{0}} \oplus \nu_{*}\left(\mathcal{O}_{\mathbb{P}_{L}^{1}}\right) \longrightarrow \nu_{*}\left(\mathcal{O}_{A}\right) \longrightarrow 0
$$

which in turn gives a long exact sequence of $K$-vector spaces

$$
0 \longrightarrow H^{0}\left(X, \mathcal{O}_{X}\right) \longrightarrow \mathcal{O}_{A_{0}} \oplus L \longrightarrow \mathcal{O}_{A} \longrightarrow H^{1}\left(X, \mathcal{O}_{X}\right) \longrightarrow 0
$$

Using that $h^{0}\left(\mathcal{O}_{X}\right)=1$, we infer that $\mathcal{O}_{A_{0}} \cap L=K$. The latter is equivalent to $L \not \subset \mathcal{O}_{A_{0}}$, because the field extension $K \subset L$ has prime degree. Being a complete intersection, the curve $X$ is Gorenstein. According to [22, chapitre IV, section 3.11, proposition 7], this implies that $\operatorname{dim}_{K}\left(\mathcal{O}_{A_{0}}\right)=$ $\operatorname{dim}_{K}\left(\mathcal{O}_{A}\right) / 2$. Now set $r=\operatorname{dim}_{K}\left(\mathcal{O}_{A}\right)$. In light of $h^{1}\left(\mathcal{O}_{X}\right)=(p-1)(p-2) / 2$, it follows that $r=(p-1)(p-2) / 2+r / 2+p-1$, whence $r=p(p-1)$. Finally, since the scheme $\operatorname{Spec}\left(\mathcal{O}_{A_{0}}\right)$ contains only one point and embeds into $\mathbb{P}_{K}^{2}$, it embeds even into $\mathbb{A}_{K}^{2}=\operatorname{Spec} K\left[U_{1}, U_{2}\right]$, whence the $K$-subalgebra $\mathcal{O}_{A_{0}} \subset \mathcal{O}_{A}$ is generated by two elements.

The situation is very simple in characteristic 2 .

Corollary 4.5. If $p=2$, then $\mathcal{O}_{A}=L$ and $\mathcal{O}_{A_{0}}=K$. 
There is more to say for odd primes. Here we have to distinguish two cases, according to the residue field of the singularity $a_{0} \in X$, which is either $K$ or $L$.

Proposition 4.6. Suppose that $p \geq 3$ and that $\kappa\left(a_{0}\right)=\mathcal{O}_{A_{0}} / \mathfrak{m}_{A_{0}}$ equals $L$. Then we have that $\mathcal{O}_{A_{0}}=K[\mu+f, g]$ for some $\mu \in L \backslash K, f \in$ $\mathfrak{m}_{A} \backslash \mathfrak{m}_{A}^{2}$, and $g \in \mathfrak{m}_{A}^{2} \backslash \mathfrak{m}_{A}^{3}$.

Proof. According to [20, Theorem 60], the projection $\mathcal{O}_{A_{0}} \rightarrow L$ onto the residue field admits a section. In other words, it is possible to embed $L=$ $\mathcal{O}_{A_{0}} / \mathfrak{m}_{A_{0}}$ as a coefficient field $L^{\prime} \subset \mathcal{O}_{A_{0}}$; however, note that such coefficient fields are not unique. By Proposition 4.4, the $K$-algebra $\mathcal{O}_{A_{0}}$ is generated by two elements, say, $h, g \in \mathcal{O}_{A_{0}}$. In case $h, g \in \mathfrak{m}_{A_{0}} \cup K$, the residue field would be $K$, a contradiction. Without loss of generality, we may assume that $h \in \mathcal{O}_{A_{0}}$ generates a coefficient field $L^{\prime} \subset \mathcal{O}_{A_{0}}$. Let $\mu \in L$ be the image of $h$ in the residue field. Inside $\mathcal{O}_{A}=L[u] /\left(u^{p-1}\right)$, we have $h=\mu+f$ for some $f \in \mathfrak{m}_{A}$. To continue, write $g=\epsilon u^{l}$ for some unit $\epsilon \in \mathcal{O}_{A}$ and some integer $0 \leq l \leq p-1$. Adding some polynomial in $h$ to $g$, we may assume that $l \geq 1$. We now check that $l=2$. Clearly, $\mathcal{O}_{A_{0}}=L^{\prime}[g] /\left(g^{d}\right)$, where $d=\lceil(p-1) / l\rceil$, such that

$$
p(p-1) / 2=\operatorname{dim}_{K}\left(\mathcal{O}_{A_{0}}\right)=p\lceil(p-1) / l\rceil .
$$

This equation implies that $(p-1) / 2 \geq(p-1) / l>(p-1) / 2-1$, which easily gives us $l=2$.

It remains to verify $f \notin \mathfrak{m}_{A}^{2}$. One way of seeing this is to use the fact that the local Artin ring $\mathcal{O}_{A}=\mathcal{O}_{\mathbb{P}_{L}^{1}} / \mathfrak{c} \simeq L[u] /\left(u^{p-1}\right)$ contains a canonical coefficient field, namely, the image of $H^{0}\left(\mathbb{P}_{L}^{1}, \mathcal{O}_{\mathbb{P}_{L}^{1}}\right)$. This gives a splitting $\mathcal{O}_{A}=L \oplus \mathfrak{m}_{A}$ of $L$-vector spaces, whence a projection pr : $\mathcal{O}_{A} \rightarrow \mathfrak{m}_{A}$. Note that this projection does not depend on any choices of the uniformizer $u$. Seeking a contradiction, we now assume that $f \in \mathfrak{m}_{A}^{2}$. Then $\operatorname{pr}\left(\mathcal{O}_{A_{0}}\right) \subset \mathfrak{m}_{A}^{2}$. On the other hand, the discussion at the beginning of Section 4 tells us that $C \subset \mathbb{P}_{K}^{2}$ is defined by a homogeneous equation of the form $\lambda U_{0}^{p}+P(\lambda) U_{1}^{p}+$ $U_{2}^{p}=0$. The preimage $a \in \mathbb{P}_{L}^{1}$ of the singular point $a_{0} \in C$ has homogeneous coordinates $\left(-P^{\prime}\left(\lambda^{1 / p}\right): 1\right)$, according to Remark 4.3. The normalization is described in Proposition 4.1 and sends $U_{0} / U_{1}$ to the element $T_{0} / T_{1}=$ $u-P^{\prime}\left(\lambda^{1 / p}\right)$, where $u=T_{0} / T_{1}+P^{\prime}\left(\lambda^{1 / p}\right)$. It follows that $u \in \mathfrak{m}_{A}$ is contained in $\operatorname{pr}\left(\mathcal{O}_{A_{0}}\right) \subset \mathfrak{m}_{A}$, a contradiction. 
Proposition 4.7. Suppose that $p \geq 3$ and that $\kappa\left(a_{0}\right)=\mathcal{O}_{A_{0}} / \mathfrak{m}_{A_{0}}=K$. Then we have $\mathcal{O}_{A_{0}}=K[v, w]$ for some $v, w \in \mathfrak{m}_{A}$ so that their classes mod $\mathfrak{m}_{A}^{2}$ are $K$-linearly independent.

Proof. Write $\mathcal{O}_{A_{0}}=K[v, w]$ for some $v, w \in \mathfrak{m}_{A}$. Then the monomials $v^{i} w^{j}$ with $i+j \geq p-1$ vanish. Since we have $\operatorname{dim}_{K}\left(\mathcal{O}_{A_{0}}\right)=p(p-1) / 2$, the monomials $v^{i} w^{j} \in R^{\prime}$ with $i+j \leq p-2$ form a $K$-basis, whence $v^{p-2}, w^{p-2} \neq$ 0 , such that $v, w \notin \mathfrak{m}_{A}^{2}$. Seeking a contradiction, we now assume that $v \equiv \alpha w$ modulo $\mathfrak{m}_{A}^{2}$ for some $\alpha \in K$. Then $v^{p-2}=\alpha^{p-2} w^{p-2}$, contradicting linear independence.

\section{§5. Abstract multiple curves}

We keep the notation from the preceding section but now reverse the situation. Fix an $L$-rational point $a \in \mathbb{P}_{L}^{1}$. Let $A \subset \mathbb{P}_{L}^{1}$ be the $(p-2)$ th infinitesimal neighborhood of $a$, and write $\mathcal{O}_{A}=L[u] /\left(u^{p-1}\right)$. Now choose a $K$-subalgebra $\mathcal{O}_{A_{0}} \subset \mathcal{O}_{A}$ and consider the resulting morphism $A=$ $\operatorname{Spec}\left(\mathcal{O}_{A}\right) \rightarrow \operatorname{Spec}\left(\mathcal{O}_{A_{0}}\right)=A_{0}$. The pushout square

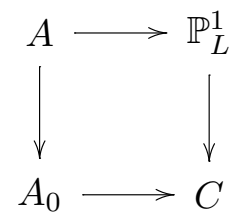

defines a proper integral curve $C$, with normalization $\nu: \mathbb{P}_{L}^{1} \rightarrow C$ and containing a unique singular point $a_{0} \in C$.

We call the subalgebra $\mathcal{O}_{A_{0}} \subset \mathcal{O}_{A}$ admissible if it takes the form described in Corollary 4.5, Proposition 4.6, or Proposition 4.7. From now on we assume that our subalgebra is admissible, and we ask whether or not the resulting curve $C$ is embeddable into $\mathbb{P}_{K}^{2}$ as a $p$-Fermat plane curve. The cohomology groups indeed have the right dimensions.

Proposition 5.1. We have $h^{0}\left(\mathcal{O}_{C}\right)=1$ and $h^{1}\left(\mathcal{O}_{C}\right)=(p-1)(p-2) / 2$.

Proof. It is easy to see that an admissible subalgebra $\mathcal{O}_{A_{0}} \subset \mathcal{O}_{A}$ satisfies the conclusion of Proposition 4.4, that is, that $\operatorname{dim}_{K}\left(\mathcal{O}_{A_{0}}\right)=p(p-1) / 2$ and $L \not \subset \mathcal{O}_{A_{0}}$. The statement in question follows from the exact sequence

$$
0 \longrightarrow H^{0}\left(C, \mathcal{O}_{C}\right) \longrightarrow \mathcal{O}_{A_{0}} \oplus L \longrightarrow \mathcal{O}_{A} \longrightarrow H^{1}\left(C, \mathcal{O}_{C}\right) \longrightarrow 0
$$

as in the proof for Proposition 4.4. 
To proceed, we have to study the behavior of $C$ under base change. If $K \subset K^{\prime}$ is a field extension, then the induced curve $C^{\prime}=C \otimes_{K} K^{\prime}$ sits inside the Cartesian and co-Cartesian diagram

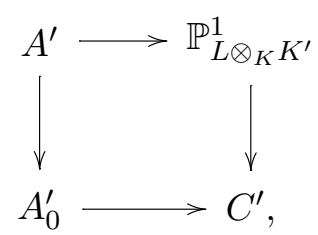

with $A^{\prime}=A \otimes_{K} K^{\prime}$ and $A_{0}^{\prime}=A_{0} \otimes_{K} K^{\prime}$. In particular, $C^{\prime}$ is nonreduced if and only if the extension field $K \subset K^{\prime}$ contains $L$. The next result implies that, under suitable assumptions, $C^{\prime}$ locally looks like a Cartier divisor inside a regular surface.

Proposition 5.2. Suppose that $L \subset K^{\prime}$. Then every closed point on $C^{\prime}$ has embedding dimension 2.

Proof. The assertion is clear outside the closed point $a_{0}^{\prime} \in C^{\prime}$ corresponding to the singularity $a_{0} \in C$. To understand the embedding dimension of $\mathcal{O}_{C^{\prime}, a_{0}^{\prime}}$, let $M, G$ be two indeterminates, and consider the $K$-algebra

$$
R=K[M][[G]]
$$

which is a regular ring of dimension 2 that is a formally smooth $K$-algebra. Therefore, it suffices to construct a surjection $R \rightarrow \mathcal{O}_{C, a_{0}}^{\wedge}$. We do this for the case that $\kappa\left(a_{0}\right)=L$, that is, that $\mathcal{O}_{A_{0}}=K[\mu+f, g]$ as in Proposition 4.6. (The other cases are similar, actually simpler.) Choose lifts $\tilde{f}, \tilde{g} \in \mathcal{O}_{C, a_{0}}$ for $f, g \in \mathcal{O}_{A^{\prime}}$, and define

$$
h: R \longrightarrow \mathcal{O}_{C, a_{0}}^{\wedge}, \quad M \longmapsto \mu+\tilde{f}, \quad G \longmapsto \tilde{g} .
$$

Note that we have to work with $R$ rather than its formal completion $K[[M$, $G]$ ], because the image $\lambda+\tilde{f}$ does not lie in the maximal ideal. Obviously, the composite map $R \rightarrow \mathcal{O}_{C, a_{0}}^{\wedge} \rightarrow \mathcal{O}_{A_{0}}$ is surjective. Now let $\lambda u^{i} \in \mathcal{O}_{C, a_{0}}^{\wedge} \subset L[[u]]$ be a monomial with $\lambda \in L$ and $i \geq p-1$. By completeness, it suffices to check that this monomial lies in $h(R)$ modulo $u^{i+1}$. Consider first the case that $i$ is even. Write $g^{i / 2}=\lambda^{\prime} u^{i}$ modulo $u^{i+1}$ for some nonzero $\lambda^{\prime} \in L$, and write $\lambda / \lambda^{\prime}=P(\mu)$ as a polynomial of degree $<p$ with coefficients in $K$ in terms of the generator $\mu \in L$. Then $\lambda u^{i}=P(\lambda+f) \cdot g^{i / 2}$ modulo $u^{i+1}$. 
Finally, suppose that $i$ is odd. Write $i=p+j$ for some even $j \geq 0$. Then $(\mu+f)^{p}=\mu^{p}+\alpha u^{p}$ modulo $u^{p+1}$ with nonzero $\mu^{p}, \alpha \in K$. Moreover, $g^{j / 2}=$ $\lambda^{\prime} u^{j}$ modulo $u^{j+1}$ for some $\lambda^{\prime} \in L$. As above, we find some polynomial $P$ of degree $<p$ with $P(\lambda)=\lambda / \lambda^{\prime}$. Then

$$
\lambda u^{i}=P(\lambda+f) \frac{(\mu+f)^{p}-\mu^{p}}{\alpha} g^{j / 2} \bmod u^{i+1} .
$$

Using that $R$ is $G$-adically complete and that $\mathcal{O}_{C, a_{0}}^{\wedge}$ is complete, we infer that $h: R \rightarrow \mathcal{O}_{C, a_{0}}^{\wedge}$ is surjective.

Proposition 5.3. Suppose that $L \subset K^{\prime}$. Then $C_{\text {red }}^{\prime}=\mathbb{P}_{K^{\prime}}^{1}$.

Proof. It suffices to treat the case $K^{\prime}=L$. The diagram (5) yields a birational morphism

$$
\nu: \mathbb{P}_{K^{\prime}}^{1}=\left(\mathbb{P}_{L \otimes_{K} K^{\prime}}^{1}\right)_{\text {red }} \longrightarrow C_{\text {red }}^{\prime} .
$$

Let $a_{0}^{\prime} \in C^{\prime}$ and $a^{\prime} \in \mathbb{P}_{K^{\prime}}^{1}$ be the points corresponding to the singularity $a_{0} \in$ $C$. It suffices to check that the fiber $\nu^{-1}\left(a_{0}^{\prime}\right) \subset \mathbb{P}_{K^{\prime}}^{1}$ is the reduced scheme given by $a^{\prime}$. In other words, the maximal ideal $\mathfrak{m}_{A^{\prime}} \subset \mathcal{O}_{A^{\prime}}$ is generated by the maximal ideal $\mathfrak{m}_{A_{0}^{\prime}}$ and the nilradical of $\mathcal{O}_{\mathbb{P}_{L \otimes_{K} K^{\prime}}^{1}}$. Let us do this in the case where the residue field of $a_{0} \in C$ is $L$, that is, $\mathcal{O}_{A_{0}}=K[\mu+f, g]$ as in Proposition 4.6 (the other two cases being similar). Then $\mathcal{O}_{A_{0}^{\prime}}$ is generated over $L$ by $\mu \otimes 1+f \otimes 1$ and $g \otimes 1$, whence also by $\mu \otimes 1-1 \otimes \mu+f \otimes 1$ and $g \otimes 1$. But the nilradical of $\mathbb{P}_{L \otimes_{K} K^{\prime}}^{1}$ is generated by $\mu \otimes 1-1 \otimes \mu$. By assumption, $f \in \mathcal{O}_{A}$ generates the maximal ideal, and this implies that $f \otimes 1 \in \mathcal{O}_{A^{\prime}}$ generates the maximal ideal modulo $\mu \otimes 1-1 \otimes \mu$, whence the claim.

If $L \subset K^{\prime}$, then $C^{\prime}$ is a nonreduced curve with smooth reduction and multiplicity $p$, and for each closed point $y \in C^{\prime}$, the complete local ring $\mathcal{O}_{C^{\prime}, y}^{\wedge}$ is a quotient of a formal power series ring in two variables over $\kappa(y)$. Loosely speaking, one may say that $C^{\prime}$ locally embeds into regular surfaces. Such curves were studied, for example, by Bănică and Forster [3], Manolache [19], and Drézet [8] and are called abstract multiple curves. Let $\mathcal{N} \subset \mathcal{O}_{C^{\prime}}$ be the nilradical. Then the ideal powers

$$
\mathcal{O}_{C^{\prime}}=\mathcal{N}^{0} \supset \mathcal{N} \supset \mathcal{N}^{2} \supset \cdots \supset \mathcal{N}^{p}=0
$$

define a filtration on the structure sheaf $\mathcal{O}_{C^{\prime}}$. The graded piece $\mathcal{L}=\mathcal{N} / \mathcal{N}^{2}$ is a coherent sheaf on $C_{\text {red }}^{\prime}=\mathcal{O}_{\mathbb{P}_{K^{\prime}}^{1}}$, and we obtain an algebra map $\operatorname{Sym}(\mathcal{L}) \rightarrow$ 
$\operatorname{gr}\left(\mathcal{O}_{C^{\prime}}\right)$. A computation in the complete local rings shows that this map is surjective, with the ideal generated by $\operatorname{Sym}^{p}(\mathcal{L})$ as kernel, and that $\mathcal{L}$ is invertible. Set $d=\operatorname{deg}(\mathcal{L})$. Using

$1-(p-1)(p-2) / 2=\chi\left(\mathcal{O}_{C^{\prime}}\right)=\chi\left(\operatorname{gr}\left(\mathcal{O}_{C^{\prime}}\right)\right)=\sum_{j=0}^{p-1}(j d+1)=d p(p-1) / 2+p$,

we infer that $\operatorname{deg}(\mathcal{L})=-1$. This shows the following.

Proposition 5.4. If we have $L \subset K^{\prime}$, then the associated graded algebra is given by $\operatorname{gr}\left(\mathcal{O}_{C^{\prime}}\right)=\bigoplus_{i=0}^{p-1} \mathcal{O}_{\mathbb{P}_{K^{\prime}}^{1}}(-i)$, with the obvious multiplication law.

We shall say that $C^{\prime}$ globally embeds into a smooth surface if there is a smooth proper connected $K^{\prime}$-surface $S$ into which $C^{\prime}$ embeds. We remark in passing that the surface $S$ is then geometrically connected or, equivalently, that $K^{\prime}=H^{0}\left(S, \mathcal{O}_{S}\right)$, because we have $K^{\prime} \subset H^{0}\left(S, \mathcal{O}_{S}\right) \rightarrow$ $H^{0}\left(C^{\prime}, \mathcal{O}_{C^{\prime}}\right)=K^{\prime}$.

TheOREM 5.5. Suppose that $C^{\prime}$ embeds globally into a smooth surface and that $p \neq 3$. Then $C^{\prime}$ embeds as a $p$-Fermat plane curve into $\mathbb{P}_{K^{\prime}}^{2}$.

Proof. We first consider the case that $L \subset K^{\prime}$, for which the assumption $p \neq 3$ plays no role. Choose an embedding $C^{\prime} \subset S$ into a smooth proper connected surface. Then $D=C_{\text {red }}^{\prime}$ is isomorphic to a projective line. By Proposition 5.4, its self-intersection inside $S$ is the number $D^{2}=1$. Set $\mathcal{L}=\mathcal{O}_{S}(D)$. The exact sequence of sheaves $0 \rightarrow \mathcal{O}_{S} \rightarrow \mathcal{L} \rightarrow \mathcal{L}_{D} \rightarrow 0$ gives an exact sequence

$$
0 \rightarrow K^{\prime} \longrightarrow H^{0}(S, \mathcal{L}) \longrightarrow H^{0}\left(D, \mathcal{L}_{D}\right) \longrightarrow H^{1}\left(S, \mathcal{O}_{S}\right)
$$

Suppose for the moment that $H^{1}\left(S, \mathcal{O}_{S}\right)=0$. Then $\mathcal{L}$ is globally generated and has $h^{0}(\mathcal{L})=3$. The resulting morphism $r: S \rightarrow \mathbb{P}_{K^{\prime}}^{2}$ is surjective, because $(\mathcal{L} \cdot \mathcal{L}) \neq 0$, and its degree equals $\operatorname{deg}(r)=(\mathcal{L} \cdot \mathcal{L})=1$, whence $r$ is birational. Moreover, the induced morphism $r: D \rightarrow \mathbb{P}_{K^{\prime}}^{2}$ is a closed embedding, and $r(D) \subset \mathbb{P}_{K^{\prime}}^{2}$ is a line. Using $D^{2}=1=r(D)^{2}$, we conclude that the exceptional curves for $r$ are disjoint from $D$. It follows that $C^{\prime}$ embeds into $\mathbb{P}_{K^{\prime}}^{2}$. Obviously, it becomes a $p$-Fermat plane curve, because $D=C_{\text {red }}^{\prime}$ becomes a line.

We now check that indeed $H^{1}\left(S, \mathcal{O}_{S}\right)=0$. For this we may assume that $K^{\prime}$ is algebraically closed. Now $K_{S} \cdot D=-3$, whence $K_{S}$ is not numerically 
effective. By the Enriques classification of surfaces, $S$ is either the projective plane or ruled. If there is a ruling $f: S \rightarrow B$, then $D$ is not contained in a fiber, because $D^{2}>0$, whence $D \rightarrow B$ is dominant, and it follows that $B$ is a projective line. In any case, $H^{1}\left(S, \mathcal{O}_{S}\right)=0$.

Finally, we have to treat the case that $K \subset K^{\prime}$ is linearly disjoint from $L$. Tensoring with $K^{\prime}$, we easily reduce to the case $K^{\prime}=K$. Choose separable and algebraic closures $K \subset K^{s} \subset \bar{K}$ and an embedding $C \subset S$ into a proper smooth connected surface $S$. Following [11, section 5], we write $\operatorname{Pic}(S / K)=$ $\operatorname{Pic}_{S / K}(K)$ for the group of rational points on the Picard scheme. According to the previous paragraph, $S_{\bar{K}}$ is a rational surface, whence the abelian group $\operatorname{Pic}\left(S_{\bar{K}}\right)$ is free of finite rank, and the scheme $\operatorname{Pic}_{S / K}$ is étale at each point. It follows that the canonical map $\operatorname{Pic}\left(S_{K^{s}} / K^{s}\right) \rightarrow \operatorname{Pic}\left(S_{\bar{S}} / \bar{S}\right)$ is bijective. Now let $[D] \in \operatorname{Pic}\left(S_{K^{s}}\right)$ be the class of $D=\left(C_{\bar{K}}\right)_{\text {red }}$. This class is necessarily invariant under the action of the Galois group $\operatorname{Gal}\left(K^{s} / K\right)$, because $p D=C_{\bar{K}}$ comes from a curve on $S$ and the abelian group $\operatorname{Pic}\left(S_{\bar{K}}\right)$ is torsion-free. We conclude that the class $[D] \in \operatorname{Pic}(S / K)$ exists, although it does not come from an invertible sheaf on $S$. However, it gives rise to a two-dimensional Brauer-Severi scheme $B$, which comes along with a morphism $r: S \rightarrow B$ that induces the morphism $r_{\bar{K}}: S_{\bar{K}} \rightarrow \mathbb{P}_{\bar{K}}^{2}$ defined above. The upshot is that there is an embedding $C \subset B$ and that the class of $C$ inside $\operatorname{Pic}(B / K)=\mathbb{Z}$ equals $p$. On the other hand, the class of the dualizing sheaf $\omega_{B}$ equals -3 . Since $p \neq 3$ by assumption, we must have that $\operatorname{Pic}(B)=\mathbb{Z}$, and consequently our Brauer-Severi scheme is $B \simeq \mathbb{P}_{K}^{2}$. It is then easy to check that $B \subset \mathbb{P}_{K}^{2}$ is indeed a $p$-Fermat plane curve.

\section{§6. Genus 1 curves}

The goal of this section is to study geometric nonreducedness for genus 1 curves. Throughout, $K$ denotes a ground field of arbitrary characteristic $p>0$. A genus 1 curve is a proper geometrically irreducible curve $X$ over $K$ with $h^{0}\left(\mathcal{O}_{X}\right)=h^{1}\left(\mathcal{O}_{X}\right)=1$. Clearly, this notion is stable under field extensions $K \subset K^{\prime}$. Since $h^{0}\left(\mathcal{O}_{X}\right)=1$, the curve $X$ contains no embedded component, such that the dualizing sheaf $\omega_{X}$ exists.

Proposition 6.1. Let $X$ be a reduced genus 1 curve. Then $X$ is Gorenstein, and $\omega_{X} \simeq \mathcal{O}_{X}$.

Proof. We have that $h^{0}\left(\omega_{X}\right)=h^{1}\left(\omega_{X}\right)=1$; hence, $\omega_{X}$ admits a nonzero section $s$. The map $s: \mathcal{O}_{X} \rightarrow \omega_{X}$ is injective, because $X$ is reduced. Hence, 
we have a short exact sequence

$$
0 \longrightarrow \mathcal{O}_{X} \stackrel{s}{\longrightarrow} \omega_{X} \longrightarrow \mathcal{F} \longrightarrow 0
$$

for some torsion sheaf $\mathcal{F}$. Using $h^{1}(\mathcal{F})=0$ and $\chi(\mathcal{F})=\chi\left(\omega_{X}\right)-\chi\left(\mathcal{O}_{X}\right)=0$, we conclude that $\mathcal{F}=0$, and the result follows.

Since $H^{2}\left(X, \mathcal{O}_{X}\right)=0$, the Picard scheme $\operatorname{Pic}_{X}$ is smooth and onedimensional, so the connected component of the origin $\mathrm{Pic}_{X}^{0}$ is an elliptic curve, a twisted form of $\mathbb{G}_{m}$, or a twisted form of $\mathbb{G}_{a}$. In other words, it is proper, of multiplicative type, or unipotent. A natural question is whether all three possibilities occur in genus 1 curves that are regular but geometrically nonreduced. It turns out that this is not the case.

THEOREM 6.2. Let $X$ be a genus 1 curve that is regular but geometrically nonreduced. Then $\mathrm{Pic}_{X}^{0}$ is unipotent.

Proof. Seeking a contradiction, we assume that the Picard scheme is not unipotent. Choose an algebraic closure $K \subset \bar{K}$, and set $Y=X \otimes_{K} \bar{K}$. Proposition 6.1 applied to $X$ yields that $Y$ is a genus 1 curve with $\omega_{Y}=\mathcal{O}_{Y}$. Let $\mathcal{N} \subset \mathcal{O}_{Y}$ be the nilradical, which defines the closed subscheme $Y_{\text {red }} \subset Y$. We first check that $Y_{\text {red }}$ is also a genus 1 curve with $\omega_{Y_{\text {red }}}=\mathcal{O}_{Y_{\text {red }}}$. The short exact sequence $0 \rightarrow \mathcal{N} \rightarrow \mathcal{O}_{Y} \rightarrow \mathcal{O}_{Y_{\text {red }}} \rightarrow 0$ yields a long exact sequence

$$
H^{1}(Y, \mathcal{N}) \longrightarrow H^{1}\left(Y, \mathcal{O}_{Y}\right) \longrightarrow H^{1}\left(Y_{\text {red }}, \mathcal{O}_{Y_{\text {red }}}\right) \longrightarrow 0
$$

Since the Picard scheme $\operatorname{Pic}_{Y}^{0}$ contains no unipotent subgroup scheme, the restriction mapping $H^{1}\left(Y, \mathcal{O}_{Y}\right) \rightarrow H^{1}\left(Y_{\text {red }}, \mathcal{O}_{Y_{\text {red }}}\right)$ is injective, which follows from [4, Section 9.2, Proposition 5], whence $h^{1}\left(\mathcal{O}_{Y_{\text {red }}}\right)=1$. Furthermore, we have that $h^{0}\left(\mathcal{O}_{Y_{\text {red }}}\right)=1$ since $Y_{\text {red }}$ is integral and $\bar{K}$ is algebraically closed. Consequently, $Y_{\text {red }}$ is a genus 1 curve, and Proposition 6.1 tells us that $\omega_{Y_{\text {red }}}=\mathcal{O}_{Y_{\text {red }}}$.

Relative duality for the inclusion morphism $Y_{\text {red }} \rightarrow Y$ yields the formula

$$
\mathcal{O}_{Y_{\text {red }}}=\omega_{Y_{\text {red }}}=\operatorname{Hom}\left(\mathcal{O}_{Y_{\text {red }}}, \omega_{Y}\right)=\operatorname{Hom}\left(\mathcal{O}_{Y_{\text {red }}}, \mathcal{O}_{Y}\right)
$$

The term on the right is nothing but the annulator ideal $\mathcal{A} \subset \mathcal{O}_{Y}$ of the nilradical $\mathcal{N} \subset \mathcal{O}_{Y}$ such that $\mathcal{A}=\mathcal{O}_{Y_{\text {red }}}$ as $\mathcal{O}_{Y}$-modules and, in particular, that $h^{0}(\mathcal{A})=1$. To finish the proof, consider the closed subscheme $Y^{\prime} \subset Y$ defined by $\mathcal{A} \subset \mathcal{O}_{Y}$. By assumption, $\mathcal{N} \neq 0$ such that $\mathcal{A} \neq \mathcal{O}_{Y}$, and therefore $Y^{\prime} \neq \emptyset$. We have an exact sequence

$$
0 \longrightarrow H^{0}(Y, \mathcal{A}) \longrightarrow H^{0}\left(Y, \mathcal{O}_{Y}\right) \longrightarrow H^{0}\left(Y^{\prime}, \mathcal{O}_{Y^{\prime}}\right)
$$


where the map on the right is nonzero, whence $h^{0}\left(\mathcal{O}_{Y}\right) \geq 2$, a contradiction.

Corollary 6.3. Let $X$ be a genus 1 curve that is regular but geometrically nonreduced. Then the reduction of the induced curve $\bar{X}$ over the algebraic closure $K \subset \bar{K}$ is isomorphic to the projective line or the rational cuspidal curve.

Proof. Set $C \subset \bar{X}$ as the reduction. Clearly, $h^{1}\left(\mathcal{O}_{C}\right) \leq 1$. We have that $C=\mathbb{P}_{\bar{K}}^{1}$ if $h^{1}\left(\mathcal{O}_{C}\right)=0$. Now assume that $h^{1}\left(\mathcal{O}_{C}\right)=1$. Then $\mathrm{Pic}_{C}^{0}$ is unipotent by Theorem 6.2. According to [4, Section 9.3, Corollary 12], this implies that the normalization map $\nu: C^{\prime} \rightarrow C$ is a homeomorphism, which means that $C$ is the rational cuspidal curve.

Acknowledgment. It is a pleasure to thank Igor Dolgachev, since stimulating discussions with him originated this research. I also thank Burt Totaro for pointing out some references, and I thank the referee for carefully reading the manuscript.

\section{REFERENCES}

[1] H. Ahmad, The algebraic closure in function fields of quadratic forms in characteristic 2, Bull. Austral. Math. Soc. 55 (1997), 293-297.

[2] L. Bădescu, Algebraic Surfaces, Universitext, Springer, New York, 2001.

[3] C. Bănică and O. Forster, "Multiplicity structures on space curves" in The Lefschetz Centennial Conference, Part I (Mexico City, 1984), Contemp. Math. 58, Amer. Math. Soc., Providence, 1986, 47-64.

[4] S. Bosch, W. Lütkebohmert, and M. Raynaud, Néron Models, Ergeb. Math. Grenzgeb. (3) 21, Springer, Berlin, 1990.

[5] N. Bourbaki, Algèbre commutative, Masson, Paris, 1983.

[6] _ Algebra II, Elem. Math. (Berlin), Springer, Berlin, 1990.

[7] R.-O. Buchweitz, D. Eisenbud, and J. Herzog, "Cohen-Macaulay modules on quadrics" in Singularities, Representation of Algebras, and Vector Bundles, Lecture Notes in Math. 1273, Springer, Berlin, 1987, 58-116.

[8] J.-M. Drézet, Paramétrisation des courbes multiples primitives, Adv. Geom. 7 (2007), $559-612$.

[9] A. Grothendieck, Éléments de géométrie algébrique, IV: Étude locale des schémas et des morphismes de schémas, II, Publ. Math. Inst. Hautes Études Sci. 24, Springer, Heidelberg, 1965.

[10] , Éléments de géométrie algébrique, IV: Étude locale des schémas et des morphismes de schémas, III, Publ. Math. Inst. Hautes Études Sci. 28, Springer, Heidelberg, 1966.

[11] _ "Le groupe de Brauer III" in Dix exposés sur la cohomologie des schémas, North-Holland, Amsterdam, 1968, 88-189.

$[12]-$ Sections hyperplanes et projections coniques (EGA V), preprint. 
[13] D. Hoffmann, "Diagonal forms of degree $p$ in characteristic $p_{2}$ " in Algebraic and Arithmetic Theory of Quadratic Forms, Contemp. Math. 344, Amer. Math. Soc., Providence, 2004, 135-183.

[14] D. Hoffmann and A. Laghribi, Quadratic forms and Pfister neighbors in characteristic 2, Trans. Amer. Math. Soc. 356 (2004), 4019-4053.

[15] J.-P. Jouanolou, Théorèmes de Bertini et applications, Prog. Math. 42, Birkhauser, Boston, 1983.

[16] J. Kollár, Extremal rays on smooth threefolds, Ann. Sci. École Norm. Sup. 24 (1991), 339-361.

[17] H. Kraft, Inseparable Körpererweiterungen, Comment. Math. Helv. 45 (1970), 110118.

[18] S. MacLane, Modular fields, I: Separating transcendence bases, Duke Math. J. 5 (1939), 372-393.

[19] N. Manolache, Multiple structures on smooth support, Math. Nachr. 167 (1994), 157202.

[20] H. Matsumura, Commutative Algebra, 2nd ed., Math. Lecture Note Ser. 56, Benjamin/Cummings, Reading, Massachusetts, 1980.

[21] S. Mori and N. Saito, Fano threefolds with wild conic bundle structures, Proc. Japan Acad. Ser. A Math. Sci. 79 (2003), 111-114.

[22] J.-P. Serre, Groupes algébriques et corps de classes, Actualités Sci. Indust. 1264, Hermann, Paris, 1975.

[23] B. Totaro, Birational geometry of quadrics in characteristic 2, J. Algebraic Geom. 17 (2008), 577-597.

Mathematisches Institut

Heinrich-Heine-Universität

40225 Düsseldorf

Germany

schroeer@math.uni-duesseldorf.de 